\title{
How an ongoing practice changes - Digitale Assistenztechnologie in der Behindertenhilfe
}

\author{
Konstantin Rink • Tristan Gruschka • Udo Seelmeyer • Gudrun Dobslaw • \\ Dominic Becking • Patrick Palsbröker
}

Eingegangen: 7. Februar 2020 / Angenommen: 1. September 2020 / Online publiziert: 1. April 2021

(C) Der/die Autor(en) 2021

Zusammenfassung Das vom BMBF geförderte Projekt Personalized Augmented Guidance for the Autonomy of People with Intellectual Impairments (PAGAnInI) ist interdisziplinär ausgerichtet und widmet sich zum einen der technischen Entwicklung eines Smartphone-gestützten Lernsystems, welches es Menschen mit kognitiven Beeinträchtigungen ermöglichen soll, sich weitgehend autonom im öffentlichen Raum zu bewegen. Zum anderen untersucht das Projekt den Implementierungsprozess dieser neuen digitalen Technologie in die Praxis der Behindertenhilfe. Mit Hilfe von Interviews, teilnehmender Beobachtung und Gruppendiskussionen wollen wir den Transformationsprozess der Praxis rekonstruieren und kritisch analysieren. Unser Projekt analysiert das Verhältnis von Technik, Akteur*innen und Adressat*innen in Bezug auf die Frage des Sozialpädagogischen und des professionellen Handelns und hat zum Ziel, sozialwissenschaftliche Grundlagenforschung, Praxisentwicklung und informationstechnische Entwicklung des Systems eng zu verzahnen.

\footnotetext{
K. Rink, M.A. $(\bowtie)$ · T. Gruschka, M.A. · Prof. Dr. U. Seelmeyer · Prof. Dr. G. Dobslaw Interaktion 1, Fachhochschule Bielefeld, 33619 Bielefeld, Deutschland E-Mail: Konstantin.rink@fh-bielefeld.de

T. Gruschka, M.A.

E-Mail: tristan.gruschka@fh-bielefeld.de

Prof. Dr. U. Seelmeyer

E-Mail: udo.seelmeyer@fh-bielefeld.de

Prof. Dr. G. Dobslaw

E-Mail: gudrun.dobslaw@fh-bielefeld.de

Prof. Dr. D. Becking · P. Palsbröker, M.A.

Fachhochschule Bielefeld, Artilleriestraße 9, 32427 Minden, Deutschland

Prof. Dr. D. Becking

E-Mail: dominic.becking@fh-bielefeld.de

P. Palsbröker, M.A.

E-Mail: patrick.palsbroeker@fh-bielefeld.de
} 
Schlüsselwörter Digitalisierung · Soziale Arbeit · Menschen mit kognitiver Beeinträchtigung · Hybride Dienstleistungen · Partizipative Forschung

\title{
How an ongoing practice changes-Digital assistance technology in disability care
}

\begin{abstract}
The project Personalized Augmented Guidance for the Autonomy of People with Intellectual Impairments (PAGAnInI), which is sponsored by the German Federal Ministry of Education and Research (BMBF), has an interdisciplinary focus. On the one hand, the project is dedicated to the technical development of a smartphone-supported learning system that is intended to enable people with cognitive impairments to move largely autonomously in public spaces. On the other hand, the project analyzes the integration process of this new digital technology into the practice of care for people with disabilities. Using interviews, participant observations and group discussions, we reconstruct and critically analyze the transformation process of the practice. Our project analyzes the relationship between technology, social workers and clients in relation to the question of social work and professional action and aims to closely interlink basic social science research, practice development and software development.
\end{abstract}

Keywords Digital transformation - Social work $\cdot$ People with cognitive disabilities $\cdot$ Hybrid social services $\cdot$ Participatory research

\section{Problemaufriss}

Die Feststellung, dass heute kaum ein Arbeitsbereich nicht vom digitalen Wandel betroffen zu sein scheint, ist schon ein Allgemeinplatz, dessen Aussage wenig über den wirklich stattfindenden Wandel preisgibt. Denn die Arbeitsfelder sind höchst heterogen von dem Wandel, den immer neue digitale Technologien mit sich bringen, betroffen: Während einige Sektoren radikale Veränderungen erfahren, gilt die personenbezogene Dienstleistungsarbeit ,,als kaum standardisierbar und automatisierbar, nicht zuletzt wegen ihres interaktiven und wissensintensiven Charakters sowie wegen der zentralen Bedeutung der Einbindung der Konsumenten für das Zustandekommen der Leistung“" (Seelmeyer und Waag 2020, S. 180).

Doch seit einigen Jahren scheint sich auch diese Gewissheit zu verflüchtigen. Weite Teile der Sozialen Arbeit bleiben nicht unberührt vom Einsatz digitaler Technologien ${ }^{1}$. ,[N]eben der sozialisatorischen Prägung von Fachkräften und Adressat_innen durch digitale Medien“" (Seelmeyer und Kutscher 2017, S. 229) wandelt sich ,zunehmend auch der Kern interaktiver Dienstleistungserbringung“ (Seelmeyer und Waag 2020, S. 180). Hinzu kommt, dass sozialpädagogische Organisationen ebenfalls tiefgreifenden Veränderungen unterliegen, wie etwa in der Standardisierung von Fällen durch Fallsoftware. Demnach stehen sowohl Organisationen, als auch Professionelle und Adressat*innen vor der Herausforderung, sich gegenüber

\footnotetext{
1 Vgl. Überblicke in Kutscher et al. (2020); Goldkind et al. (2019); Watling und Rogers (2012).
} 
der Transformation, die die Gesellschaft in den letzten Jahren durch die Einführung digitaler Informations- und Kommunikationstechnologien erfahren hat, zu verhalten. Um dabei nicht zu passiven Statist*innen degradiert zu werden, müssen sie sich dem Wandel stellen und aktiv mitgestalten.

Einige Handlungsfelder der Sozialen Arbeit haben die technologischen Veränderungen zum Anlass genommen, um innovative Neuerungen zu diskutieren und einzuführen, während wiederum in anderen Feldern eine deutliche Skepsis gegenüber Digitalisierungsentwicklungen erkennbar wird. Die Behindertenhilfe gehört wohl zu letzteren (Kreidenweis 2018). Nicht allein die Webseiten der Einrichtungen sind oft unübersichtlich und in unverständlicher Fachsprache verpackt, sondern auch die Tätigkeit der Behindertenhilfe gilt für viele der darin Arbeitenden als ausschließlich menschliche Praxis (Kreidenweis 2018). Gleichzeitig deutet aber vieles darauf hin, dass neue Assistenztechnologien, Dokumentationssysteme oder Serviceroboter auch in Einrichtungen der Behindertenhilfe früher oder später eingesetzt werden. Diesen Prozess gilt es aktiv durch das Einbringen eigener Vorstellungen zu gestalten.

Aber nicht allein die Praxis hat so ihre Schwierigkeiten mit den sich rasant vollziehenden Veränderungen. Auch im Kontext der empirischen Forschung spielen neue technologische Artefakte und deren Bedeutung nur eine marginale Rolle. In Anbetracht des stattfindenden Wandels und der geringfügigen Forschung dazu existiert ein ,dringender Forschungsbedarf bezogen auf die Transformationsdynamiken, die sich im Zusammenspiel von digitaler Technik, Akteuren, organisationalem und wohlfahrtsstaatlichem Kontext ergeben“" (Seelmeyer und Kutscher 2017, S. 235).

\section{Projektzielsetzung und -design}

Genau an dem Schnittbereich von aktiver Technikgestaltung, Implementierung und Transformationsforschung setzt das Forschungsprojekt Personalized Augmented Guidance for the Autonomy of People with Intellectual Impairments (PAGAnInI) an, das u. a. an Vorarbeiten zur Implementation von neuer Technologie im Feld der Behindertenhilfe anknüpft (Becking und Vaudt 2014). Von besonderem Interesse für PAGAnInI sind die veröffentlichten Ansätze von João Ramos et al. (2013) und Robbie Fryers et al. (2018), die ein Navigationssystem für Smartphones für Menschen mit kognitiven Einschränkungen entwickelten, welches die Mobilität im öffentlichen Raum erleichtern soll. Bei den bestehenden Systemen handelt es sich allerdings um reine Navigations-Apps. PAGAnInI geht mit seinem adaptiven Ansatz weit über die bisherigen Systeme hinaus. Denn es werden drei Ziele im Rahmen des Projektes verfolgt:

a) Zum einen wird in partizipativer Zusammenarbeit mit Menschen mit kognitiven Beeinträchtigungen ein Smartphone-gestütztes Lernsystem entwickelt, mit Hilfe dessen sich diese Personengruppe möglichst selbstständig im öffentlichen Raum bewegen können soll. Durch Funktionen für ein individuelles Trainings-Arrangement ist das System PAGAnInI weit mehr als eine Navigations-App. Ausgehend vom individuellen Lernstand und den Bedürfnissen wird das System eingerichtet und lernt mit. Es ist das Ziel, dass Menschen mit kognitiven Beeinträchtigungen 
ihre Wege - wie etwa den Arbeitsweg - autonom zurücklegen können; am Ende auch ohne die App.

b) Zum Zweiten geht es darum, auf organisationaler und professioneller Ebene den Implementierungsprozess der App in die Praxis der Sozialen Arbeit kritisch zu begleiten und zu analysieren. Ziel ist es, Fragen im Laufe der Forschung zu beantworten wie: Wie wird die App in (bestehende) fachliche Strukturen eingebettet? Welche dominanten Muster ergeben sich auf der Ebene der Organisation? Wie verändern sich sozialpädagogische Organisationen im Kontext der Einführung digitaler Technologien?

c) Schließlich besteht ein weiteres Arbeitsziel darin, über die Evaluation der Zusammenarbeit zwischen Fachkräften, Wissenschaftler*innen und Adressat*innen aussagekräftige Informationen zu gewinnen, wie eine partizipative Forschung mit Menschen mit kognitiven Beeinträchtigungen gestaltet werden kann.

Insgesamt verortet sich das Projekt damit auch im Feld der Teilhabeforschung. Dazu gehört, dass der Projektverbund (verschiedene Disziplinen der FH Bielefeld) eng mit den Praxispartner*innen bei der Entwicklung, Durchführung und Auswertung zusammenarbeitet. Zudem wird, um die Belange von Menschen mit kognitiver Beeinträchtigung vollumfänglich zu berücksichtigen, ein Gremium mit Vertreter*innen der genannten Personengruppe eingerichtet, das kontinuierlich alle Schritte des Prozesses begleitet.

\section{Forschungsmethodik}

In einem ersten Schritt werden zunächst die Ausgangsbedingungen und bisherigen Mobilitätspraktiken der Menschen mit kognitiven Beeinträchtigungen mittels leitfadengestützter Interviews und teilnehmender Beobachtung rekonstruiert. Erhoben werden sowohl die individuellen Mobilitätspraktiken der Menschen im öffentlichen Raum (im Sinne eines Mitgehens ${ }^{2}$ ) wie auch deren Praktiken im Umgang mit Smartphone und anderen digitalen Technologien. Ebenfalls sollen mit Hilfe von Interviews Fachkräfte und Expert*innen in den Einrichtungen zu den rahmenden organisationalen Abläufen befragt werden.

Während die App in die Praxis eingeführt wird, werden wir in einem weiteren Schritt die durch die Einführung eines solchen Systems einhergehenden Anpassungen vorhandener Prozesse und eventuell auch Strukturen teilnehmend beobachten und in Gruppendiskussionen reflektieren lassen. Zeitgleich werden Interaktionen zwischen Fachkräften und Menschen mit kognitiven Beeinträchtigungen mittels Videokamera aufgezeichnet und ausgewertet.

\footnotetext{
2 Wir nehmen Bezug auf die Arbeiten Mitgehen als Methode von Margarethe Kusenbach (2008).
} 


\section{Perspektiven}

Ziel ist es, in dem geplanten Projekt sozialwissenschaftliche Grundlagenforschung, Praxisentwicklung und die informationstechnische Entwicklung des Systems eng zu verzahnen. Im Ergebnis soll nicht nur ein Smartphone-gestütztes Lernsystem zur Verfügung stehen, das die individuelle Teilhabe am gesellschaftlichen Leben effektiv unterstützt und bei deren Entwicklung die Menschen, für die es gedacht ist, umfassend mit eingebunden wurden, sondern auch eine neue Praxis in der Behindertenhilfe hergestellt und kritisch analysiert werden.

Funding Open Access funding enabled and organized by Projekt DEAL.

Open Access Dieser Artikel wird unter der Creative Commons Namensnennung 4.0 International Lizenz veröffentlicht, welche die Nutzung, Vervielfältigung, Bearbeitung, Verbreitung und Wiedergabe in jeglichem Medium und Format erlaubt, sofern Sie den/die ursprünglichen Autor(en) und die Quelle ordnungsgemäß nennen, einen Link zur Creative Commons Lizenz beifügen und angeben, ob Änderungen vorgenommen wurden.

Die in diesem Artikel enthaltenen Bilder und sonstiges Drittmaterial unterliegen ebenfalls der genannten Creative Commons Lizenz, sofern sich aus der Abbildungslegende nichts anderes ergibt. Sofern das betreffende Material nicht unter der genannten Creative Commons Lizenz steht und die betreffende Handlung nicht nach gesetzlichen Vorschriften erlaubt ist, ist für die oben aufgeführten Weiterverwendungen des Materials die Einwilligung des jeweiligen Rechteinhabers einzuholen.

Weitere Details zur Lizenz entnehmen Sie bitte der Lizenzinformation auf http://creativecommons.org/ licenses/by/4.0/deed.de.

\section{Literatur}

Becking, D., \& Vaudt, S. (2014). Assistenz-Systeme. Hilfe per Smartphone. Sozialwirtschaft, 2, 38-39.

Fryers, R., Holzer Saad, T., Kelly, K., \& Dinsmore, J. (2018). Report defining the needs of stakeholders for a wayfinding platform for individuals with intellectual disabilities and their carers. Dublin: Trinity College.

Goldkind, L., Wolf, L., \& Freddolino, P. (Hrsg.). (2019). Digital social work. Tools for practice with individuals, organizations, and communities. New York: Oxford University Press.

Kreidenweis, H. (2018). Digitalisierung ändert nichts - außer alles. Wirkungen auf Gesellschaft, Soziale Arbeit und Berufliche Rehabilitation. Die berufliche Rehabilitation: Zeitschrift zur beruflichen und sozialen Teilhabe, 2, 148-157.

Kusenbach, M. (2008). Mitgehen als Methode. Der „Go-Along“ in der phänomenologischen Forschungspraxis. In I. J. Raab, M. Pfadenhauer, P. Stegmaier, J. Dreher \& B. Schnettler (Hrsg.), Phänomenologie und Soziologie: Theoretische Positionen, aktuelle Problemfelder und empirische Umsetzungen (S. 349-358). Wiesbaden: VS.

Kutscher, N., Ley, T., Seelmeyer, U., Siller, F., Tillmann, A., \& Zorn, I. (Hrsg.). (2020). Handbuch Soziale Arbeit und Digitalisierung. Weinheim: Beltz Juventa.

Ramos, J., Anacleto, R., Novais, P., Figueiredo, L., Almeida, A., \& Neves, J. (2013). Geo-localization system for people with cognitive disabilities. In J. Pérez, et al. (Hrsg.), Trends in practical applications of agents and multiagent systems. Advances in intelligent systems and computing (S. 59-66). Wiesbaden: VS.

Seelmeyer, U., \& Kutscher, N. (2017). Mediatisierte Praktiken in der Sozialen Arbeit. Empirische Zugänge zu institutionellen Arrangements zwischen Subjekt und digitalen Medien. In D. Hoffmann, F. Krotz \& W. Reißmann (Hrsg.), Mediatisierung und Mediensozialisation: Prozesse - Räume - Praktiken (S. 229-245). Wiesbaden: VS. 
Seelmeyer, U., \& Waag, P. (2020). Hybridisierung personenbezogener sozialer Dienstleistungen. In N. Kutscher, T. Ley, U. Seelmeyer, F. Siller, A. Tillmann \& I. Zorn (Hrsg.), Handbuch Soziale Arbeit und Digitalisierung (S. 180-190). Weinheim: Beltz Juventa.

Watling, S., \& Rogers, J. (2012). Social work in a digital society. London: SAGE. 